\title{
Germanica
}

\section{Es passiert - Spuren neuer epischer Poesie}

Quelque chose (se) passe. Traces d'une nouvelle poésie lyrique

\section{Thomas Althaus}

\section{(2) OpenEdition \\ Journals}

Édition électronique

URL : http://journals.openedition.org/germanica/1467

DOI : $10.4000 /$ germanica. 1467

ISSN : 2107-0784

Éditeur

Université de Lille

\section{Édition imprimée}

Date de publication : 30 décembre 1997

Pagination : 33-49

ISBN : 977098426320321

ISSN : 0984-2632

Référence électronique

Thomas Althaus, «Es passiert - Spuren neuer epischer Poesie », Germanica [En ligne], 21 | 1997, mis en ligne le 05 juin 2012, consulté le 06 octobre 2020. URL : http://journals.openedition.org/germanica/ 1467 ; DOI : https://doi.org/10.4000/germanica.1467

Ce document a été généré automatiquement le 6 octobre 2020.

(C) Tous droits réservés 


\title{
Es passiert - Spuren neuer epischer Poesie
}

Quelque chose (se) passe. Traces d'une nouvelle poésie lyrique

\author{
Thomas Althaus
}

1 Als Walter Benjamin seit dem Ende der Zwanziger Jahre das Passagen-Werk mit der Absicht konzipiert, in da und dort vorbeiführender Reflexion durch die "Analyse des kleinen Einzelmoments den Kristall des Totalgeschehens zu entdecken $»^{1}$, dringt alsbald über das Maß des Beabsichtigten die allegorische Konzeption in die Behandlung des Gegenstandes ein. Das Notieren wird auf seine Weise ergriffen vom « Magnetismus der nächsten Straßenecke ». Die «neue Lethe, die den Asphaltstrom durch Passagen rollt», der "anamnestische Rausch, in dem der Flaneur durch die Stadt zieht $"^{2}$, nehmen das Schreiben mit und machen in dessen ständiger Fortsetzung, als Ablenkung durch Neues, das Entstehen des Textes unmöglich. Damit scheitert das Passagen-Werk aber auch an den ästhetischen Möglichkeiten des Problematisierten. Die Wahl des Gegenstandes war für Benjamin entscheidend mitbestimmt von der morbiden Sphäre, der Abblätterung Pariser Passagen des 19. Jahrhunderts, worin ihm die Unwesentlichkeit des Phänomens erschien. Die Passage de l'Opéra war schon nicht mehr da. Was aber als Text entstand oder nicht entstand, ist von so entscheidender Modernität, daß mit der Erstveröffentlichung des Konvolutes 1982 einer neuen, ebenso extendierenden Lyrik die Bahn gebrochen wird, gerade einer Lyrik. Deren Modell war doch spätestens seit den Sechziger Jahren der kleine, förmlich zusammengezogene Text (Erich Arendts «Inseln der Worte»). Eine solche Konzentration des lyrischen Ausdrucks hat jetzt per se einen amimetischen Charakter. Die Abwendung zur nächsten Straßenecke ist der natürliche Ausdruck unseres Selbst geworden. "Denn nur Geschöpfe der Fahrt sind wir / und unsere Gestalt ist Fluktuation. » « Geh aber weiter, [...] / hör nicht auf zuviel Bewußtsein. Besser, du achtest // jetzt auf die redenden Leute $»^{3}$. Also sind wir am Ende ? Das läßt sich so nicht sagen. 


\section{Guten Tag}

Etwas sehr Merkwürdiges, eine Art Wörter-, Themen- und Reflexionsbegrüßung durchzieht die Gedichte des Bandes passim von Peter Waterhouse aus dem Jahr 1986: "Realistischer Stadtstraßengruß an den Leser: Guten Tag", "Guten Tag Hölle ", "Guten Tag Unbemerktes », "Guten Tag Zunge ", "Guten Tag erdenkbare Grade», "Guten Tag Herr Briefträger ", "Guten Tag immerhin ", "Guten Tag Sprache », «Guten Tag Licht », « Guten Tag. Viele Wörter " ${ }^{4}$... Das sind gleichzeitig schon wieder Abschiede. Der Leser, die Gegenstände der Darstellung, die Sprache des Gedichts und noch das darstellende Bewußtsein werden von dieser Bewegung des Passierenden erfaßt : « Ich bin es, ich, guten Tag » (59). Jede Vorstellung, die passiert, macht den Text seinerseits zu Passierendem. Wie Vorübergehende Vorübergehende wahrnehmen, kommen der Text und seine Gedanken aneinander vorbei. Eine Neugierde ist da, vielleicht auch eine Bekanntschaft aus früherer Zeit, ähnlich kurz und zufällig wie jetzt diese. Das Flüchtige des Großstadtlebens dringt auf neue, radikale Weise in die Sphären lyrischer Intimität, bis in die letzten Reservate, in denen der poetische Modernismus immer noch prägnant zu sein gedachte. Manchmal geht es einfach nur darum, sich schnell durchzudrängen («Entschuldigung» [45], «Verzeihung, Verzeihung [...] Verzeihung» [50]). Was im nächsten Augenblick passiert, ist kaum vorauszusagen : «Achtung Dachrinne, achtung Mäuseleben / achtung Pilot Seele, Speichelkapitän, Wortsänger, Schuhbewohner / Augenöffner, Großmutter Haut [...] achtung Blaulicht » (78). Eigentlich bleibt das Gedicht unter seinen Vorstellungen ein Fremder.

3 Ein erstes Begrüßen markiert die Bedingungen bezugslos gewordener Existenz und verweist damit auf eine geschichtliche Herkunft der Haltlosigkeit. Das war nicht immer so. Aber gleichzeitig wird in spruchhafter Wendung auf den Anfang des Philosophierens als Ausgangspunkt des lyrischen Verfahrens zurückgegriffen. Das ist so, seitdem es Denken in der abendländischen Tradition überhaupt gibt : «Alles flieht. Die Hintergründe sind nicht mehr / unsere Gründe. Ganz vorne stehen wir, im / unmittelbaren nichts, guten Tag. » (8) Identität hat mit der nie sehr weit reichenden Stabilisierung von Kontexten zu tun. Es gibt sie letztlich nur als ineinander geschobene, zur Deckung gebrachte Momente der Wahrnehmungsflucht. Demnach entsteht der Horizont für eine negative Beschreibung des Wechsels erst durch die Zuordnung von Augenblicken des Insistierens im Wechsel selbst : « 0 , immer wieder ist blau blau.» (12) «Jetzt sind wir wir. Das liegt aber nur am Zeitpunkt. "(70) Also ist nichts künstlicher als die gefügte Realität. Ihre Konstruktion resultiert aus Nennungen, die ausdrücklich auch dann noch notwendig sind, wenn 'etwas' den Namen behalten soll, den es bisher hatte : "Wir sind nichts, wir / haben eine Geste des Fortgehens. Gefragt sagen wir : / In der Wiese ist gemeint die Wiese / mit dem Tisch ist gemeint der Tisch » (43). Zugleich zwingt aber jede Referenz in eine aporetische Situation. Was durch sie gelingt, mindert den auf Fortgang und Vorstellungswechsel gerichteten Wahrheitsanspruch des Gedichts :

[...] nichts hält zusammen passim muß ich sagen, es ist alles gesagt, nichts ein Atem, ein Name, wo sind wir jetzt

kein Name, die Finger, die Hose, der Sessel ein Flug : Das ist bald gesagt. (45)

4 Jede Feststellung wird konterkariert von der Satzbewegung, in der sie nur einen Augenblick lang gelten kann. Die Satzbewegung ist das intensivste Korrelat des 
Unzustandes, damit zugleich das einzig bleibend Authentische. Aber darin eilt auch eine Aufhebung der anderen nach. Ohnedies folgt das Wort dem Wort in einem Maße assoziativ, daß schon die Fortsetzung die Aufhebung des Gedankens sein könnte (aber auch die Aufhebung noch die Fortsetzung). Verfahrenskritische Argumente dagegen sind tatsächlich erkenntniskritische Argumente dafür. Die Willkür und Unverläßlichkeit der sprachlichen Operation folgt aus der prinzipiellen Kontingenz der Dinge und des Ich. Wer ist denn schon dieser Waterhouse ? Im passim Begegnenden das einmal mit «achtung Mäuseleben » Angekündigte, ein Echo und Reim auf «Maus », aber « wasserlos » « im Grunde », ein Buchstaben- und Worträtsel, nach dorthin, zum Namen auflösbar, aber auch nach hierhin, zur Maus unseres Jahrhunderts :

Wir sind die Silbe Maus, und das hat Gründe. Wir essen Käse

und denken großzügig. Hiermit ist schon manches gesagt

obgleich wir sehr sprachlos sind. Wir haben eine deutliche Nase. Diese

richtet sich zum Himmel und atmet viel. Im Grunde

ist jetzt alles gesagt. Im Grunde will heißen : Unten, dort

wo die Mäuse gehen, die Silben, die stillen Käsehändler

die explosive Landschaft, wo wir brennen ohne zu brennen

ertrinken wasserlos, man uns würgt als keiner den niemand

die Messer der Katzen uns reißen, jetzt sagt man Maus

geradeheraus, und das klingt beiläufig, endgültig, schön. 0

es ist etwas geschehen, ich habe zwei Augen

ich stehe auf Beinen, nachgezählte Schuhe, Hose, Hemd. Wer

hat solches schon gesehen? Hier ist die Grenze, jetzt sagen wir Maus.

Maus.

Das genügt. Zu sehen ist nichts. Ganzes Bewußtsein. Wovon handelt das?

So geht es weiter. Eine Art Türeinschlagen. Eine Art Tagesende.

Eine Art letztes Stockwerk. Gedichtende. Standbildstarre.

Hier wird offensichtlich gewartet. Im Warten sind wir erkennbar. Allerdings

ist das Warten ein Nicht-Warten. Die Bushaltestellen sind nur

die nach oben gekehrten Keller. Die dunklen Gänge

machen eine helle Stadt. Die Nacht ist ein Tag ohne Licht so leuchtend. Wo

wir sind, sind wir nicht. Es ist schön

ein Schatten im Strahl zu sein. Wer strahlt?

Nichts ist geschehen, nicht zu vergessen die zwei Augen

die Beine, nachgezählte Schuhe, Hose, Hemd, Himmel. Maus, das genügt

und genügt doch wieder nicht. (44)

Die textinitiierenden Vorstellungen sind gerade solche, bei denen eine abstruse lexikalische Verschiebung den Zufall und das Willkürliche der Formulierung als unüberwindbar zeigt. Ein Stück weit führt das Gedicht dann aber doch bedeutungserschließend voran, fast noch einmal in Formen lyrischer Argumentation, und sofort geht das assoziative Moment der Reflexionsfindung an die Stringenz der Implikationen verloren. Das schafft dem Gedicht in seinem Bedeutungsaufbau festere Kriterien. Nur ist es die Frage, ob darin noch ein Gelingen gesehen werden darf. In der Bindung des wortspielhaften Moments konturiert sich sukzessive die Person als eine bestimmte, unfrei geworden, der Möglichkeiten des Anders(denkbar)seins verlustig und dazu als eine von den « Katzen » des Unnennbaren abgehetzte Kreatur unter einem für sie leeren Himmel. Davor schützt auf dem Gedanken weg dann auch nicht mehr die Berufung des Normalen, die Vergewisserung des Subjekts als Nicht-Maus. Das ist kaum noch zu glauben («ich habe zwei Augen, ich stehe auf Beinen, nachgezählte Schuhe, 
Hose, Hemd. Wer / hat solches schon gesehen?»). Alles zeigt sich im Verfolg der begonnenen Erwägung auf einmal anders ( Die Bushaltestellen sind nur / die nach oben gekehrten Keller. Die dunklen Gänge / machen eine helle Stadt. »), das Gedicht registriert an sich selbst das Ende einer weiter nach oben, statt so nach unten gerichteten Transformation («Eine Art Tagesende. / Eine Art letztes Stockwerk. Gedichtende. Standbildstarre. »). Es ist nur gut, daß die Bestimmung letztlich nicht genügt, daß ihr auch wieder zu entkommen ist und der allegorische Zugriff nicht festzuschreiben ver- mag. Das Subjekt wird wieder aus dem Zwang des Gedankens herausgelöst. Das beginnt im Rekurs auf die prinzipielle Möglichkeit des Passierens schon an der Stelle, an der es nicht mehr weiterzu- gehen scheint, als brachialer Akt katachretischer Fortführung (« So geht es weiter. Eine Art Türeinschlagen.»). Und wie die lichte Welt oben, über den Kellern der Mäuse, deutbar ist als Sein in der Nacht, bei allen Zeichen des Tages, so ist umgekehrt die dunkle Existenz unten doch auch noch, mit neuer Willkür des Ansetzens, deutbar als Sein im Hellen, bei allen Zeichen des Dunkels, mit diesen nämlich nur als «Schatten im Strahl». Und es wird darauf geachtet, daß alles ganz mitkommt, «nicht zu vergessen die zwei Augen / die Beine, nachgezählte Schuhe, Hose, Hemd, Himmel », der Himmel also auch.

Das Vertrauen auf die Möglichkeiten des Gedichts wird paradox getragen von der Skepsis gegenüber den Möglichkeiten des Gedichts. Es hat seine Dimensionen, die Behauptung seiner Freiheit nachgerade in der Provisorik seiner Klärung, mit der es die eigenen totlaufenden Konkretionen doch noch zu passieren vermag. Das ist bei aller fehlenden Situierung lyrischer Erkenntnis wenigstens auch ein Losschreiben von kontextabhängiger Sicherung. «Im hinausgeschickten Fuß begann das Gehen, das sich ohne Götter im anderen / Fuß fortsetzte» (30). Vielleicht ist darin noch die alte Wegmetaphorik in ihrer langen Tradition als Indikator textleitender reflektierter Diskursivität erinnert; gesucht wird aber nach den negativen Derivaten der Sukzession. «Was haltlos ist / macht eine besondere Fahrt. » (17) Die Linearität des Textes ist angewiesen auf das Entbundensein der Reflexion, «wenn alles da wäre / als Vögel, als Züge, als nichts, als Wort, einer wie wir / wäre ungeschickt und stolperte. So lernt man gehen. » (62) Schon die Versbildung unterliegt der Fragwürdigkeit, mit der jede geschlossene Formulierung zu dezidierter Wahrnehmung führt und dezidierte Wahrnehmung zu Gewißheiten, gegen die dann nur noch das Weiterkommen helfen kann. Die lyrische Formation des Textes ist eine aufhaltende Reminiszenz an den überlieferten Typus Gedicht. Durch sie hindurch wird eine episierende Diktion rekonstruiert. Ein rhapsodisches Fortsetzen, in abstraktester Form als Durchzählung (" 19 gedreht heißt 18,18 / dreht sich weiter, 17 fällt in den sechzehnten Sinn / fünfzehnte Stille » [26], « Erstes Herz [...] Zweites Herz [...] Drittes Herz [...] Viertes Herz [...] das fünfte Herz. Schönstes Herz.» [27]), ist das Mittel gegen die negativen Konsequenzen je und je. Kann ein Gedicht ohne dies, auf welche Weise immer, in seinem Bestimmen heute noch wahr vor sich selbst und zugleich nicht negativ sein? In der Lyrik von Waterhouse ist da, deutlich unter den Bedingungen der Lyrik von Celan, Ausweglosigkeit verbürgt, das " unmittelbare nichts ", in dem wir "ganz vorne stehen [...], guten Tag. » (8) Hier hilft nur noch ein Weitergehen der Lyrik, ein Spaziergang als Himmelskunst :

Guten Tag Kunst : So muß man beginnen. Warum ? Im Grüßen

bleiben die Übergänge sichtbar. Die Grundlage des Grüßens Heißt :

Es gibt nur Übergänge, die gute Welt ist ein einziges Sagen :

Guten Tag, und kommt herüber 
als Dinge (erstens : Die Maispflanze ist ein einziges Grüßen

wie es nach oben wächst ; zweitens : Der Holzboden ist ein einziger Guter Tag

von unten; drittens verweist auf viertens

man kann das alles zählen) und

es gibt die stumme Nacht auch (brennt in ihrer Weise

in den Augen, rauscht in den Ohren

macht unsem Atem so schwarz). Die Grundlage der Nacht heißt :

Es gibt nur Untergänge : Der grußlose Gang des Gelösten

in die Tiefe - verfluchte Tiefe. Wir

sind nicht tief, der eigene Abgrund heißt Fuß

im Kopfstand werfen wir diesen Abgrund Richtung Götter

zehn Engel die Zehen und jeder Engel sagt :

Guten Tag Kunst des Kopfstands. [...] (19)

7 Stabilisiert sich der Gedichtprozeß zu spezifischer Referenz, ist zwar hinter das Flüchtige des Passierenden zu gelangen. Das kehrt aber nur Gegenwahrheiten hervor, "Übergänge " als "Untergänge ", die Stelle "des Grüßens» nimmt dann "der grußlose Gang des Gelösten / in die Tiefe - verfluchte Tiefe » ein. Ein Höherreichen gibt es nur in der Abwendung solcher Negation. Wichtig ist die mit dem entbotenen Gruß angegebene Richtung in die Sprache hinein. Vor weiterer Erkundigung ist schnell zum nächsten Gruß überzugehen. Wie oberflächlich das bleibt, so notwendig bleibt es aber auch : ist es doch mit uns selbst außerhalb dessen nur die Nacht „hinter uns“, und erst « im guten Übergang » sind und haben wir etwas, vorher nicht. Die Identität des movierenden Bewußtseins liegt allein in den textdurchziehenden Fragen nach seinem Aufenthalt und seinem Fortkommen (im Augenblick der Äußerung der Frage) : "Wo sind wir jetzt? », «Wo / sind wir jetzt?» (7), « Wohin?» (14), «Wo sind wir? ? (19), «Wo wollten wir hin?» (20)... In der Bewegung verbleibt die durch den eigenen Körper mögliche sinnliche Erfahrung als einzige Realität. "Der eigene Abgrund heißt Fuß». Und ein solcher Abgrund, nur ein solcher ist im Kopfstand nach oben zu verschieben, die Büchner-Celan-Kehre nun als Gewinn von "guter Welt“. Freilich geht es nicht ohne die restlose Nominalisierung der Vorstellungsgehalte durch die beiherlaufende Rede, die jegliche Willkür leidet, auch diese. Größtes Problem und einzige Lösung: es kann dann auf einmal der ganze Raum neu ausgemessen werden. "Die gute Welt ist ein einziges Sagen: / Guten Tag». Alle Dinge wachsen statt zum Licht zum Gruß der Passanten auf. In ein neues natura loquitur führt damit der Weg. Aus den Reduktionen gewinnt diese Lyrik am Ende nicht weiterzudenkender Entwicklung den Impetus des Anfangs zurück. Natürlich kann auch die "Maus» in solche Bewegung gebracht werden. In der Bewegung ist keine Denomination endgültig. "Maus» kann « Mais », «Maus. Mais » werden, das Subjekt in neuer, kurz geltender Position. Aber nirgendwo deutlicher als dort hängt die Sprache mit dem großen Anspruch - « ein einziges Grüßen / wie es nach oben wächst »- an der ganz kleinen Rede en passant, an der letzten Depravierung : «Der Mais in die Höhe zum Wetter : wie sehe ich aus?» (84)

Im Lichte des Verfahrens erscheint die lyrische Moderne als Vorbereitung der neuen dezentrierten Textform : "Alles im Gedicht war im Übergang. Nichts im Gedicht war bei sich. Das festumrissene Sich, Ich, Du, Es hörte ich an keiner Stelle des Gedichts. Das Gedicht stand da ohne Fundament, ohne Verabredung, ohne Gewißheit. » (Waterhouse zu Andrea Zanzottos Silicio, Carbonio, Castellieri) - «Sprechen über ein Ereignis in der Ortlosigkeit [...] ohne Statik [...] im Zustand von - her [...] Ich sah die Worte aus der Inhaltsposition gleiten. » (Waterhouse zu Paul Celans Stimmen) - «Ich hörte in dem Gedicht den wiederkehrenden Übergang. [...] Ich hörte die Behauptungslosigkeit, die 
Unwirklichkeit, die Erweiterbarkeit, das Nicht-Selbst, die Aufrufbarkeit» (Waterhouse zu Carl Rakosis Yaddo) 5 . Dabei wird für die dezentrierte Textform gleichzeitig durch die Vereinbarung von Lyrik und Prosa zum «Gedicht. Roman » auf beider Ursprung aus epischer Poesie zurückgewiesen. Waterhouse schreibt Das Klarfeld Gedicht von 50 Seiten : « Zuerst braucht es die Ferne / die Ferne, lange Wege, denen man sich nähert / erzählend, zeigend, verwerfend, lachend und bekümmert » - «Passant Passant / Ende der Vermessenheit $»^{6}$.

\section{What happened?}

1986 geht auch die Lyrik von Jürgen Becker in ein sequentielles Dichten über. Das Gedicht Die Scheune, das den Band Odenthals Küste abschließt, enthält 91 Passagen, die noch lesbar wären als eine zyklische Konstellation, dränge nicht gleichzeitig eine Ästhetik der Reihung in den Satzbau vor. Und die einzelne Wahrnehmung erhält erst durch ihr Nicht-Identisches, ihren Anstoß zu weggleitender Erinnerung eigenes Gewicht : « mit einer rappligen Sense schlägst du / den Hohlweg frei (und mitten durch / mein Gedächtnis geht diese Schneise / ins Jahr 44: zwei Lightnings griffen / den Pflaumenbaum an) ${ }^{7}$. Es gibt einen Ort der Erinnerung, die Scheune, prädestiniert zu idyllischer Beschreibung. So ist der Ort aber nicht beschreibbar, weil die Wörter, die es leisten sollen, von dem Kontext ihrer sonstigen Verwendung im Jahrhundert der Kriege nicht mehr abzulösen sind (man muß nur 'Schneise' und 'Schlagen' sagen, schon ist die Flugschneise und der Einschlag der Bomben da). Die annähernde Unmöglichkeit, das Katastrophale nicht zu sagen, ruft die Erinnerung wach, der bei allem alles Schlimme einfällt. Wo es mit dem Wort gelingen könnte, nun doch einmal das Gefahrlose zu denken, steht also die Erinnerung dagegen, und gegen die Erinnerung doch auch an Gefahrloses steht das Wort. Schon inkonsequent ist es, beide als unbeschädigte Kategorien einzuräumen. Wie es denn auch gar nicht mehr anders denkbar sein kann, weist das, was Wort und Erinnerung einerseits ermöglichen, andererseits auf die Zerstörungen an ihnen selbst zurück. « Spur / durch ein Gedächtnis; die Fläche ward / leer, [...] die Schrift riß» (463). Sicher, es war immer noch, auch in der lyrischen Moderne, Sprachlosigkeit in einen Unsagbarkeitstopos $\mathrm{zu}$ wenden. Aber die Vorstellung vom Anschluß an lyrikgeschichtliche Entwicklungen, womöglich als günstiges Verfügen über literarische Mittel, umgeht das Problem, wie jetzt gleichsam auf einem Ritt über den Bodensee letzte Intensität des Ausdrucks besser nicht zur Darstellungsintention gehört. "Indes die Birnen, / Rauchsäulen, Wiesen sichtbar wachsen, und / nichts ist passiert" (474), grenzt es an ein Wunder, daß an «Rauchsäulen" vorbeizukommen war und sich nicht eine schreckliche Bedeutung entband. Da wird der Ausdruck der langen Weile, «nichts ist passiert ", zum Satz der Beruhigung, daß es gerade noch gut gehen konnte. Die Perspektive liegt in einem Darüber-hinweg. Mit naturlyrischer Konkretion ist es ohnehin vorbei, gleich zu Beginn in einer programmatischen Abweisung: keine Botschaften des Regens mehr, so lange läßt sich gar nicht zuhören, und zu gefährlich wäre es. Denn « nie Trommler war der Regen / ein leichtes Gewehr » (463).

Eine Erfahrung müßte also möglich sein, die dem Trauma entkommt, indem sie den Schutz der Verdrängung nicht preisgibt. Ihr Paradigma wird im Gedicht die mediale und jetzt bereits virtuelle Welt der «Programme » jeder Art « auf einem Bildschirm» : «Die nächste Geschichte / hat schon niemand erlebt ; ganz von allein / entsteht sie auf 
einem Bildschirm » (470), « die imaginäre Schrift, die jetzt / übern Bildschirm huscht » (483), «jederzeit der Wechsel / zum unbekannten Programm» (484), "die einzige Gewißheit, daß / der Austausch nützlicher, sinnloser Informationen / nicht stockt. » (486) Wiewohl in so kritischer Bezugnahme die Erinnerungsarbeit des Gedichts gegen die Defizienz medialer Vergegenwärtigung gestellt ist, bleibt diese Arbeit dennoch an deren Formen verwiesen, an den kritisierten schnellen Weg ins Nächste. Er bestimmt den Text zur Auflösung tradierter Formationen als Gedicht. Er mindert als spezifischer Modus der Übergänglichkeit in der sicheren Aussicht des Fortkommens die Bedrückung durch das Geschehene. Das Schlimmste läßt sich wegzappen. Vor allem werden die Konturen differenter, gebrochener Einheiten (Einheiten ?) der Vorstellung verschliffen. Da ist die eine Wahrheit das Schwinden der Intensität von Erfahrungsmomenten und die andere der Gewinn einer Möglichkeit zur Anreihung und Konstellierung für den verfügbaren Rest. Umbrüche werden Schnittstellen, Kola Module. Der Text frequentiert seine Themen on line: der Speicher der Scheune wird in wortspielhafter Übertragung zum Speicher der Daten ihrer Geschichte ( $«$ es ist heiß; die Daten knistern / im Speicher.» [478]). Mit den Daten wird ein Transfer oder mehr schon ein Surfen begonnen, das durch ihre Menge gleiten läßt. Im gleichen Vorgang, in dem die Destrukturierung den Text zerstört, entbindet sie früheste textuelle Qualitäten. Eine neue epische Breite wird bemerkbar, ebenso eine Rückfindung in die Memorialfunktionen des Rhythmus unter Aufgabe prosodischer Qualitäten. Dann geht es schon nicht mehr nur um den Weg zurück in die Erinnerung. Vielmehr baut sich das, was erinnert wird, für den Blick nach hinten nun selbst aus seinen Anfängen bereits als Prozeß ständiger Wahrnehmungsverschiebung auf, der das Markante synchronisiert. Und dieser Prozeß hat schließlich, wieder nach vorn gedacht, am Zeitpunkt des Jetzt auch nur noch einen Zeitpunkt des Vorübergleitens :

$$
\text { [...] Im sinkenden Licht }
$$

dieses Tages taucht eine Zuversicht auf, die frische Spur

meines Gehens, die aus dem Kreis der Verzögerungen

herausführt, [...]

[...] Unendliches Gehen,

das eine fortgesetzte Beschreibung verspricht,

sonst nichts verlangt, keine Augenzeugen im Dunkel (493f.)

11 So geht es weiter, alles passiert. In Das englische Fenster (1990) kommentiert eine Parenthese das Gedichtverfahren, so daß der Blick auf das Ganze einrutscht in die bewegten Reflexionen :

$$
\text { etcetera ; kein }
$$

Bildervergleich dauert länger als ein paar Minuten,

in denen, aus Bruchstücken zusammengerafft, ein

riesiges Panorama erscheint, das

bei näherem Hinsehn rasch wieder zerfällt in

Fensterausschnitte, Figuren zwischen Landkartenzeichen,

Zimmerfluchten und Straßenzüge,

Satzteile, Schlagerfetzen, Gelächter und Schluchzer

hinter den angelehnten Türen ; etcetera $-{ }^{8}$

12 Natürlich schieben sich mit der Parenthese auch deren Kontexte durch das Text «fenster» (« dieses Fenster, eine Passage für / die unterschiedlichsten Arten der Gegenwart » [587]). Das englische Fenster - words for Windows, winwords.Es ist eineweitere Affinität der Gedichtstruktur zur Textverarbeitung, daß der Versfall den 
Eindruck erweckt, das alles geschähe tatsächlich schon mehr von oben nach unten als von links nach rechts.

Schwer zu entscheiden bleibt, ob das Verfahren noch zu Wahrnehmungen führt, rechnet es doch die Wahrnehmungsminderung als ästhetische Qualität ein. In genau dieser Beziehung ist es ein Nachahmungsvorgang, in genau dieser aber auch die Infragestellung der Möglichkeiten des Gedichts : «Die Mühen der Rekonstruktion / bleiben sinnlos; zuviel war passiert ${ }^{9}$. Eigentlich folgt aus beidem, aus dem mimetischen Anspruch (wenn es denn einer ist) und aus den Schwierigkeiten der Darstellung, daß die Kriterien der neuen epischen Poesie lediglich der Verlegenheit geschuldet sind, das Textmaterial in einem intensiveren Sinn nicht mehr als Gedicht zusammenbringen zu können. Doch setzen die Texte an jeder Stelle fortschreitender Überbrückung immerhin zu einem Versuch punktueller Erfahrung an. Die weite Führung bildet die Vorstellungsflut ab, in der die Reflexionsinsel Gedicht sonst wohl zu gewinnen war, während jetzt eben alles passiert. Konzentriert sich aber der Text trotzdem auf eine stellenbezogene Klärung, ist nichts mehr da, was sich klären ließe. Alles passiert nicht anders als etwas einzelnes bereits in sich selbst. Auf die gleichzeitig höchst irritierende wie völlig gewöhnliche Weise von Leben heute ist es seiner Sache nach absent :

$$
\text { plötzlich }
$$

im Scheinwerferlicht. Wir stoppen und steigen aus.

« What happened? « Der Mann weiß nicht, warum

er blutend da liegt, neben seinem Motorrad; wir

können nicht sagen, was passiert ist -

(Später

versuche ich diesen Moment zu erklären, als etwas

Erlebtes, das nie zu erklären ist... es gibt

keine Erinnerung, es gibt keine Zeugen ; es ist nur

etwas geschehen, das wie alles Geschehen

eine genaue und endlose Vorgeschichte hat

und nun... abbricht, weitergeht, sich verzweigt und

verflechtet mit werweißwelchem Geschehen ? [...] $)^{10}$

14 Die Wahrnehmung der Personen, Dinge, Ereignisse wird zunehmend dominiert von der ihres Vorübergehens. Im Ansatz ist das ein Problem des Epischen überhaupt. Weil Aufhaltungen nicht möglich sind, gehören zum epischen Duktus seit je versus iterati, Formeln einer epischen Wiederholung (wie das homerisch 'geflügelte Wort') als Indikator für den Erhalt von Identität. In der Lyrik Beckers wechselt spätestens mit dem Gedichtband Foxtrott im Erfurter Stadion (1993) das Motiv der Wiederholung vom Gegenstand zur Formel des Passierens selbst hinüber :

[...] Riesiges, das langsam

verschwindet... es ist schon verschwunden, noch vor

einem Dekret. Er wiederholt es ; er kann erst gehen, wenn

nichts mehr passiert. Man wird ihn vermissen ${ }^{11}$.

Was wiederholt er? Das Riesige, das verschwindet, oder nicht doch schon den Satz über dessen Verschwinden? Und bald - das ist angekündigt - bezieht die Wahrheit des Satzes auch den Sprechenden ein. Zu wiederholen und zu merken ist, oft genug in wortgenauer Aufnahme, daß etwas «passiert». Das bedingt «eine vorläufige Topographie. / Du kannst sie verwischen. Du kannst sie / verändern, bis eine Serie entsteht, bis wir erreichen / die Ufer der Wiederholung» (710). Bildet der Fluß der Dinge selbst die «Ufer der Wiederholung », bleibt etwas paradoxerweise nur dadurch, 
daß alles flieht. Wohl gibt es sehr deutliche Eindrücke, die nicht zu ändern sind. Aber das betrifft die Katastrophen mit Endgültigkeit, auf die nichts mehr folgt. Die prinzipielle Angst davor ist den Sätzen noch dort abzunehmen, wo sie versichern wollen, daß sie unbegründet sei : "Nichts // ist passiert in der Parkebene unten » (664). $\mathrm{Zu}$ jemandem auf einer Straße im Krieg wird gesagt: " du kannst jetzt passieren. » (651) Und der, der nicht abtransportiert wurde, kann zu sich selbst sagen : "die Züge, in denen ich saß, / kamen immer irgendwo an, wo / nichts weiter passierte. » (694f.) So enthält die endlos anfügende Rede aus sich Gewährleistungen, die im Dargestellten keineswegs sicher sind. Dabei kann jedoch der einfache Umstand des Literarischen für sich noch nicht vor dem Real werden der Angst schützen. Traumatische Erfahrungen holen ihr Opfer immer wieder ein, es sei denn, dieses ist schneller im raschen Durchgang seiner Wiederholungen :

[...] Vom Ufer, wo zwischen den Büschen die Angler sitzen, feuern plötzlich Maschinengewehre; wenig passiert; die Uferstraße bleibt weiter passierbar, und junge Leute mit schwarzen Tüchern betreten die Diskothek. (658f.)

Solange eine Geschichte sich aus der anderen entwickelt, tritt nach jüdischer Vorstellung der Tod nicht ein. Und in der Projektion nach vorn kommt etwas wie Erwartung hinzu. Ein neuer Text Beckers Aus dem Journal der Wiederholungen beginnt und endet so: "Nächste Seite, vielleicht passiert noch etwas [...] kann sein, / die nächste Seite weiß mehr $»^{12}$.

\section{Weiter geht's}

In Michael Buselmeiers Ich rühm dich Heidelberg gibt es die Augenblicke, mit denen manches bleibt in Nacht verloren: " $\mathrm{Ab}$ und $\mathrm{zu}$ stürzte einer / auf einen vorbeifahrenden Schleppkahn / brach sich den Halswirbel / oder platschte auf den Bauch / von der Strömung rasch abgetrieben »; "Kinder auf Schlittschuhen / eines bricht ein / versinkt strampelnd / zwischen gefrorenen Algen $»^{13}$. Nur der Cicerone, der viel und zu viel redende Fremdenführer im «langen Gedicht» $(14,65)$ von über 70 Seiten kommt daran mit notwendig gewordener Diffusität noch vorbei. Er zeigt nicht die Stellen und nicht die Stätte, sondern die Stadt, mögliche Wege durch sie hindurch. Dabei trifft der Blick der Lektüre so schnell auf ein nächstes, wie er auf dieses trifft: «Zwerg Perkeo / Kleiner Muck im Großen Faß treibend / Blumenpeter in Wiesloch 1940 / mit einer Giftspritze / um den Lebensrest gebracht... » (20) Das muß alles gesagt sein. Aber wo anfangen, wo aufhören? Die Geschichte der Heimat ist die schreckliche Aufhäufung von Ortlosigkeiten, die der Cicerone geschwätzig durchläuft. Er ist selbst ein schaulustiger Passant, einer von denen, die sich schnell wegdrücken, wenn der Anblick unerträglich wird. Schon der elegische Ton ist ihm zu viel, will er sich doch gerade wieder in die Flucht schlagen lassen :

Ach Cicerone!

Was wollt ihr von mir... (76)

18 Niemand muß noch von dem äußerst Problematischen eines solchen Bewußtseins überzeugen, - müßige Hinweise, wo die Alternativen fehlen. Es ist sich selbst die einzige Alternative: nur mit seiner Fortsetzung geht's weiter. Jeder Vers hat im nächsten, der folgt, den letzten Rest möglicher Verheißung, bei Werner Söllner :

Endstation es geht weiter sage ich mir es geht es

geht weiter es hört nicht auf hier war dein Ziel 
nicht dein Irrtum zum wievielten Mal war dein Ziel

deine Flucht $[. . .]^{14}$

Es folgen, in der neuen Form episierend, auf den Vers der Vers, der Vers, der Vers... Solange es weitergeht, geht es eben weiter. " Dann bleibt nichts, nach dem Verlust / dieser Struktur, als eine Passage $\aleph^{15}$. Das Bedauern enthält aber zugleich die Möglichkeit des neuen Gedichts vom wunderbaren Auf gehobensein in dem, was passiert. Elke Erb nennt es die Unterkunft in einer Flut :

als ich das überdenke, in der Totale,

allein hier diesen Nachmittag unter dem Himmel,

auf die Nahtstelle richte den Blick, die Passage,

da gleitet, gleich einer Antwort, unter die Augen,

so daß sie nur noch zuzusehn brauchen,

unterrichtend lautlos, doch wundersam

ein Flügel her, wie ihn die Engel haben,

und fügt sich an einem Menschenleib

- und Unterkunft finden

demnach die Intervalle, die Wartezeiten,

Schnitte und Staue, Entbehrungen, Teile, Stillstände, Wechsel

in unaufhörlicher Wellenflut ${ }^{16}$.

Mit vor Schreck erstarrtem Gesicht sieht Benjamins Engel der Geschichte zurück : wie sich vor den Menschen die Trümmer ihrer Zukunft aufbauen, in die er selbst rücklings weiter hinausfliegt. Das ist der Luxus einer eschatologischen Perspektive, die wir uns nicht erlauben können. Wir müssen ja noch hindurch. Das, was wir Fortschritt nennen, ist unser Entkommen nach vorn. Da wächst uns mit den Möglichkeiten des Passierens ein Flügel an, nicht wirklich um zu fliegen, sondern zur Bergung unter dem eigenen Fittich.

Der "ganze heutige Weltzustand ", schließt Hegel die Diskussion der Gattung ab, füge sich nicht " wahrhaft epischen Darstellungen ", einerseits der " prosaischen Ordnung ", andererseits der "Umwälzungen" (Revolutionen) wegen ${ }^{17}$. Man darf aber nicht übersehen, daß Hegels Einrede gegen das Epos in ihren literaturgeschichtlichen Dimensionen überschritten wird von dem Anspruch seines Philosophierens überhaupt. Sein Philosophieren unterliegt nämlich selbst einer Idee von Epos, «dem absoluten Epos [...], dessen Held der Menschengeist, der Humanus sein würde, der sich aus der Dumpfheit des Bewußtseins zur Weltgeschichte erzieht und erhebt $»^{18}$. Das wäre die Erfindung einer "Weltgeschichte", die ihren Namen verdiente, auf dem Wege einer Philosophie als Epos. So liegt im epischen Durchgang « höchste Handlung des Geistes ». Positionsloses Denken kann produktiv werden, ja recht besehen nur dieses. Wo sind wir, und was wissen wir schon? Deshalb muß als eine Qualität gewonnen werden, was als katastrophaler Unzustand die Texte vorantreibt und nicht halten läßt. Daß dies dem, der jetzt zu Antworten will, nicht genügen kann, ist richtig. Richtig ist aber auch, daß mit der Dynamisierung des Textes eine Statuierung des Lesers einhergeht. Während sich der Leser durch den Text bewegt, nimmt er «das Geschehen einer Handlung » wahr, « welches sich an uns vorüberbewegt $»^{19}$. In diesem Passieren erfährt die Einrede des Klagenden, es fehle eine Perspektive, die implizite Erwiderung, dies eben sei sein Problem. Da er stehen bleibt, hat er nichts zu hoffen. Wohl gilt weiterhin Achim von Arnims große Klage über den « Zweck des ganzen mühevollen Lebens, sich 
so leise wie möglich neben einander wegzuschieben ». Ach, es kann so aber doch auch « der größere Theil der Welt, eine fremde Atmosphäre, durch unsere Luft [...] hindurch gehen [...], für uns unschwer, für uns unwarm, keine Macht über uns habend, als unsre Furcht davor. $»^{20}$.

\section{NOTES}

1. Walter Benjamin : Das Passagen-Werk. Hrsg. von Rolf Tiedemann. 2 Bde. Frankfurt a.M. 1983. Bd. 1. S. 575. N 2, 6.

2. Ebd. Bd. 2. Pariser Passagen II. S. 1053 u. 1055.

3. Botho Strauß : Diese Erinnerung an einen, der nur einen Tag zu Gast war. Gedicht. Mit einer Nachbemerkung von Martin Walser. München, Wien 1992. S. 24u.S. 30f.

4. Peter Waterhouse : passim. Gedichte. Reinbek 1986. S. 12, 15, 18, 28, 50,58,78, 80 u. 82. Weitere Zitate im fortlaufenden Text.

5. Peter Waterhouse : Sprache Tod Nacht Aussen. Gedicht. Roman. Reinbek 1989. S. 38, $86 f ., 145$ u. 156.

6. Peter Waterhouse : Das Klarfeld Gedicht. Berlin 1988. S. $16 f$.

7. Jürgen Becker : Odenthals Küste. In : J.B., Die Gedichte. Frankfurt a.M. 1995. Die Scheune. S. 463-494, hier S. 477. Weitere Zitate im fortlaufenden Text.

8. Jürgen Becker : Das englische Fenster. In : J.B., Die Gedichte, a.a.O. S. 565-639, hier S. 575. Weitere Zitate im fortlaufenden Text.

9. Jürgen Becker: Das Gedicht von der wiedervereinigten Landschaft. In : J.B., Die Gedichte, a.a.O. S. 495-563, hier S. 501.

10. Jürgen Becker : Das englische Fenster, a.a.O. S. 585. Weitere Zitate im fortlaufenden Text.

11. Jürgen Becker : Foxtrott im Erfurter Stadion. In : J.B., Die Gedichte, a.a.O. S. 641-710, hier S. 662 .

12. In : Jahrbuch der Lyrik 1995/6. Poesie der Poesie. Hrsg. von Christoph Buchwald und Joachim Sartorius. München 1995. S. 13f.

13. Michael Buselmeier : Ich rühm dich Heidelberg. Poem in sechs Gesängen. Heidelberg 1996. S. 35 u. 38.

14. Werner Söllner : Kopfland. Passagen. Gedichte. Frankfurt a.M. 1988. Es geht weiter. S. 96.

15. Ebd. Passagen. S. 109-115, hier S. 112.

16. Elke Erb : Unschuld, du Licht meiner Augen. Gedichte. Göttingen 1994.S. 93f.

17. Georg Wilhelm Friedrich Hegel : Werke. Bd. 15 : Vorlesungen über die Ästhetik III. Auf der Grundlage der Werke von 1832-1845 neu edierte Ausgabe. Redaktion Eva Moldenhauer und Karl Markus Michel. Frankfurt a.M. 1970. S. 414.

18. Ebd. S. 356.

19. Ebd. S. 356.

20. Des Knaben Wunderhorn. Alte Deutsche Lieder. Gesammelt von L.A. v. Arnim und Clemens Brentano. Studienausgabe in 9 Bänden hrsg. von Heinz Rölleke. Bd. 1. Stuttgart, Berlin, Köln, Mainz 1979. Von Volksliedern. S. 406-442, hier S. 411 u. 437. 


\section{RÉSUMÉS}

Sous l'influence des Passages de Benjamin se constitue dans les années 80 un nouveau lyrisme de la décentration. Les textes de Peter Waterhouse et Jürgen Becker en viennent à faire de l'évitement leur caractère distinctif: absence de concentration, perception distraite, changement, attitudes de détour. Les détracteurs de ce lyrisme verront sans doute le trait essentiel de ces textes dans la fuite. La seule manière, pour ce monde de passants, de se protéger contre son manque de substance est de ne pas en prendre conscience, de glisser sur lui comme il glisse sur toute chose. Mais alors, la concrétion lyrique n'aboutira qu'à une aporie et débouchera sur le néant. Or, à condition qu'il y ait conscience du présent comme mouvement incessant, on voit apparaître une nouvelle écriture narrative qui commence à investir l'absence de références comme liberté, et ainsi, par une sorte de miracle salvateur, crée une perspective à partir du vide.

Unter dem Einfluß von Benjamins Passagen-Werk bildet sich in den achtziger Jahren eine neue dezentrierte Lyrik aus. Die Texte von Peter Waterhouse und Jürgen Becker erheben das Vermiedene zum Kriterium: eine spezifische Unkonzentriertheit, halbhörige Wahrnehmung, Wechselhaftigkeit, Gesten der Abwendung. Polemische Einrede dürfte darauf bestehen, daß den Texten nichts wichtiger ist als ihr eigener schneller Verlauf. Die Passanten-Welt kann sich vor ihrem Substanzlosen allein dadurch schützen, daß sie dessen nicht inne wird, dies wie alles einfach übergeht. Dann läßt aber umgekehrt die lyrische Konkretion nur noch aporetische Schlüsse auf das Nichts zu. Hingegen tritt unter den Bedingungen des Bewußtseins der Gegenwart im unentwegten Fortgang ein neuer epischer Duktus hervor, der Bezugslosigkeit als Freiheit einzusetzen beginnt und so aus dem Mangel wie in einem biblischen Rettungswunder eine Perspektive macht.

\section{AUTEUR}

\section{THOMAS ALTHAUS}

Universität Münster 